\title{
Hsa_circ_0038646 promotes cell proliferation and migration in colorectal cancer via miR-331-3p/GRIK3
}

\author{
HAIPENG DU, ZHIGUO HE, FUMEI FENG, DAMING CHEN, LEI ZHANG, JINGZHEN BAI, \\ HUIGUO WU, ENKUN HAN and JIANSHENG ZHANG
}

\author{
Department of Gastrointestinal Surgery, Tianjin Baodi Hospital, Tianjin 301800, P.R. China
}

Received August 1, 2019; Accepted January 21, 2020

DOI: 10.3892/ol.2020.11547

\begin{abstract}
Increasing evidence supports the essential roles of circular RNAs (circRNAs) and microRNAs (miRNAs/miRs) in different types of human cancer. For example, hsa circ_0137008 functions as a sponge for mi-338-5p and inhibits the malignant phenotype in colorectal cancer. Furthermore, hsa_circ_RNA_0011780 downregulates FBXW7 by targeting miR-554a and suppressing the progression of non-small cell lung cancer. Thus far, only a single report has identified that the miRNA miR-331-3p exerts a pivotal effect on human colorectal cancer (CRC) evolution. However, both the upand downstream regulatory mechanisms of miR-331-3p are unclear. In the present study, it was predicted via bioinformatics analysis that the circRNA, hsa_circ_0038646, and the glutamate receptor ionotropic kainate 3 (GRIK3) gene contain binding sites that can interact with miR-331-3p. Thus, hsa circ_0038646/miR-331-3p/GRIK3 may be a novel therapeutic pathway for CRC. Reverse transcription-quantitative PCR and western blotting analyses were performed, as well as cell proliferation, luciferase reporter and Transwell migration assays. Hsa_circ_0038646 was overexpressed in both CRC cells and tissues, and this aberrant expression was positively related with increasing tumor grade. Knockdown of hsa_circ_0038646 significantly weakened human CRC cell proliferation and migration. It was shown that hsa_circ_0038646 can sponge miR-331-3p to suppress its expression, and that suppression of miR-331-3p can reverse the effects of hsa_circ_0038646 inhibition in CRC cells. It was determined that GRIK3 is a downstream target of miR-331-3p, and that hsa_circ_0038646 could increase the levels of GRIK3 by suppressing miR-331-3p in CRC cells. Restoring GRIK3 expression rescued the weakened CRC cell proliferation and migration following
\end{abstract}

Correspondence to: Mr. Jiansheng Zhang, Department of Gastrointestinal Surgery, Tianjin Baodi Hospital, 8 Guangchuan Road, Baodi, Tianjin 301800, P.R. China

E-mail: zhangjianshengmm@163.com

Key words: colorectal cancer, hsa_circ_0038646, microRNA331-3p, glutamate receptor ionotropic kainate 3, proliferation, migration hsa_circ_0038646 knockdown. The present study indicated that hsa_circ_0038646 functions as a tumor promoter in CRC by increasing GRIK3 expression via sponging of miR-331-3p. The hsa_circ_0038646/miR-331-3p/GRIK3 axis may be a novel therapeutic and diagnostic target of CRC.

\section{Introduction}

Colorectal cancer (CRC) is a familial malignancy of the alimentary canal (1). CRC is the second most common tumor of the gastrointestinal tract, the third most frequent malignancy and the fourth most common cause of cancer-associated mortality worldwide $(2,3)$. The metastasis and recurrence rates of CRC are high, and its morbidity and mortality are increasing each year $(4,5)$. According to the histopathologic malignant degree grade of the World Health Organization (WHO) criteria, CRC can be divided into low (I and II) and high (III and IV) grades (6). Research into the pathobiology and underlying molecular mechanisms of CRC metastasis is limited (7). Further exploration of these molecular mechanisms is required.

Increasing evidence has indicated that microRNAs (miRNAs/miRs) regulate the occurrence and development of a variety of cancer cell types (8). miRNAs can modulate cell proliferation, differentiation, apoptosis and carcinogenesis by combining with the $3^{\prime}$ untranslated region (3'-UTR) of target mRNAs in cancer (9-11). miR-331-3p, located on $12 \mathrm{q} 22 \mathrm{n}$ and a member of the miR-331 family, functions as a tumor suppressor in CRC; marked downregulation of miR-331-3p in $\mathrm{CRC}$ is linked to increased cell proliferation and decreased apoptosis $(12,13)$. This aberrant expression of miR-331-3p is also linked to cell proliferation and migration in various other cancers, such as acute lymphocytic leukemia, lung cancer, glioblastoma, gastric cancer, prostate cancer, non-small cell lung cancer and pancreatic cancer (14-20). The functions of miR-331-3p in CRC cell migration, as well as the up- and downstream regulatory mechanisms of miR-331-3p, are unclear.

Circular RNAs (circRNAs) are competitive endogenous RNAs which modulate gene expression and biological function via miRNA sponging $(21,22)$. CircRNAs are highly expressed in multiple tumor tissues, including CRC $(23,24)$. CircRNAs participate in cancer-associated physiological and pathological processes, such as cell proliferation, migration and invasion, 
cell cycle progression, metastasis and carcinogenesis (25). Numerous studies show that circRNAs or long non-coding RNAs promote the progression of cancer by sponging miR-331-3p (26-28). Bioinformatics analysis predicted the presence of a binding site between hsa_circ_0038646 and miR-331-3p (26-29). In the present study, bioinformatics analysis was used to predict the binding sites for miR-331-3p in hsa_circ_0038646 and glutamate receptor ionotropic kainate 3 (GRIK3). To the author's best knowledge, no reports regarding hsa_circ_0038646 have been published to date. GRIK3 is shown to be a novel oncogenic factor in different types of cancer, including breast cancer (30) and gastric cancer (31). Thus far, the interaction between GRIK3 and miR-331-3p in $\mathrm{CRC}$ has not been reported.

In the present study, the expression and function of hsa_circ_0038646 and miR-331-3p in CRC were assessed, and the role of the hsa_circ_0038646-miR-331-3p-GRIK3 axis in CRC progression was determined.

\section{Materials and methods}

Human CRC tissue collection. Human CRC tumor tissues and adjacent normal control tissues were acquired from 62 enrolled patients with CRC who were diagnosed based on pathology, and had undergone curative surgery resection at Tianjin Baodi Hospital between May and October 2018. The cohort of patients with CRC was comprised of 25 females and 37 males, aged 52-73 years, with an average age of $59.48 \pm 6.36$ years. According to the Tumor Node Metastasis (TNM) classification standard of the Union for International Cancer Control (32), the cohort included TNM stage I ( $n=20)$, TNM stage II $(n=23)$, TNM stage III $(n=12)$ and TNM stage IV $(n=7)$. None of the patients had received anticancer treatments such as radiotherapy or chemotherapy prior to surgery. Based on WHO grading (6), the CRC tumor samples were divided into low- and high-grade groups. The present study was approved by the Clinical Ethical Committee of Tianjin Baodi Hospital and written informed consent was provided by all participants prior to the study start.

Cell culture. The human CRC cell lines SW480, HT29, DLD-1, SW620 and HCT116, as well as the normal human colon epithelial cell line NCM460, were provided by the Cell Bank of Type Culture Collection of Chinese Academy of Sciences. After cells were tested and authenticated via STR profiling, SW480 and SW620 cells were seeded in Leibovitz's L-15 medium (Gibco, Invitrogen; Thermo Fisher Scientific, Inc.). HT29 and HCT116 cells were grown in McCoy's 5A medium (Gibco, Invitrogen; Thermo Fisher Scientific, Inc.) with $10 \%$ fetal bovine serum (FBS; Invitrogen; Thermo Fisher Scientific, Inc.). DLD-1 cells were cultured in RPMI 1640 medium (Invitrogen; Thermo Fisher Scientific, Inc.) containing 5\% FBS, and $1 \%$ penicillin and streptomycin (Invitrogen; Thermo Fisher Scientific, Inc.). Notably, all cells were cultured in a humidified atmosphere of $5 \% \mathrm{CO}_{2}$ at $37^{\circ} \mathrm{C}$. Experiments were performed after $72 \mathrm{~h}$ of incubation, when the cells achieved $60-70 \%$ confluency.

Cell transfection. SW620 and HCT116 cells were seeded into 24 -well plates at a density of $5 \times 10^{4}$ for $24 \mathrm{~h}$ at $37^{\circ} \mathrm{C}$. When the cells reached $70-80 \%$ confluence, they were transfected with $120 \mathrm{nM}$ small interfering (si)RNAs, with the following sequences: hsa_circ_0038646, 5'-GUAUGGACUCAUCCA CCAGGGGA-3', and NC, 5'-GGGUAUUCACAUACCCGG GCAGA-3'; and GRIK3, 5'-GGUCUCUGGGCCAUUCAG GGGAU-3', and NC, 5'-GUGCUUGGGCACCUCGGUAUG GA-3'. Recombinant plasmids of pcDNA3.1-hsa_circ_0038646 and pcDNA3.1-GRIK3 (120 nM) were purchased from Shanghai GenePharma Co., Ltd., which were cloned into the pcDNA3.1 plasmid and the corresponding empty pcDNA3.1 plasmid (Invitrogen; Thermo Fisher Scientific, Inc.), which was used as the negative control. miR-331-3p mimics (120 nM) (5'-GCCCCUGGGCCUAUCCUAGAA-3') and mimics NC (5'-UUCUCCGAACGUGUCACGUTT-3'), miR-331-3p inhibitors (120 nM) (5'-UUCUAGGAUAGGCCCCAGGG GC-3') and inhibitors NC (5'-CAGUACUUUUGUGUAGUA CAA-3'), as well as the corresponding negative controls (NCs) were purchased from Applied Biosystems; Thermo Fisher Scientific, Inc., and performed for at least $5 \mathrm{~h}$ at $37^{\circ} \mathrm{C}$ with Lipofectamine ${ }^{\circledR} 2000$ transfection reagent (Invitrogen; Thermo Fisher Scientific, Inc.), according to the manufacturer's instructions. After $72 \mathrm{~h}$, cells were harvested.

Cell proliferation assay. Following $48 \mathrm{~h}$ of transfection and at $70 \%$ confluency, the cells were incubated for an additional 24, 48, 72 or $96 \mathrm{~h}$. Then, $12 \mu \mathrm{l}$ of Cell Counting Kit-8 (CCK-8) solution (7sea Biotech Co., Ltd.) was added into each well for 24,48 and $72 \mathrm{~h}$, respectively, and maintained at $37^{\circ} \mathrm{C}$ for $2 \mathrm{~h}$. For quantification, cell viability was detected at $450 \mathrm{~nm}$ using the SUNRISE Microplate Reader (Tecan Group, Ltd.), as previously described (33).

Cell migration assay. A Transwell assay was performed to assess cell migration. After cultured SW620 and HCT116 cells reached $80 \%$ confluence, cells $\left(5 \times 10^{4}\right)$ were plated in the upper chamber of Transwell plates in serum-free medium without FBS, for 24 h. Leibovitz's L-15 medium (for SW620) (Gibco; Thermo Fisher Scientific, Inc.) or McCoy's 5A medium (for HCT116) (Gibco; Thermo Fisher Scientific, Inc.) supplemented with $10 \%$ FBS were pre-added in the lower chambers, respectively. and the temperature were maintained at $37^{\circ} \mathrm{C}$ for $24 \mathrm{~h}$. Subsequently, cells in the upper chamber were discarded, and the migratory cells were fixed with $4 \%$ formaldehyde for $30 \mathrm{~min}$ at room temperature, prior to staining with $0.5 \%$ crystal violet solution for $30 \mathrm{~min}$ at room temperature. The migratory cells were blindly counted in five randomly selected fields, under a light microscope (magnification, x200) and using Image J software (version 1.42; National Institutes of Health). The experiment was performed in triplicate.

Reverse transcription-quantitative polymerase chain reaction $(R T-q P C R)$. Total RNA was extracted from human CRC tissues or cells with TRIzol ${ }^{\circledR}$ reagent (Invitrogen; Thermo Fisher Scientific, Inc.), according to the manufacturer's instructions. cDNA was synthesized using the RevertAid ${ }^{\mathrm{TM}}$ First Strand cDNA Synthesis kit (DBI Bioscience) at $95^{\circ} \mathrm{C}$. NanoDrop $^{\text {TM }}$ ND 1000 microspectrophotometer (NanoDrop Technologies; Thermo Fisher Scientific, Inc.) was used to quantify the extracted RNA. RT-qPCR was carried out on an ABI 7900 system (Applied Biosystems; Thermo Fisher Scientific, Inc.) using SYBR Select Master Mix (Applied 

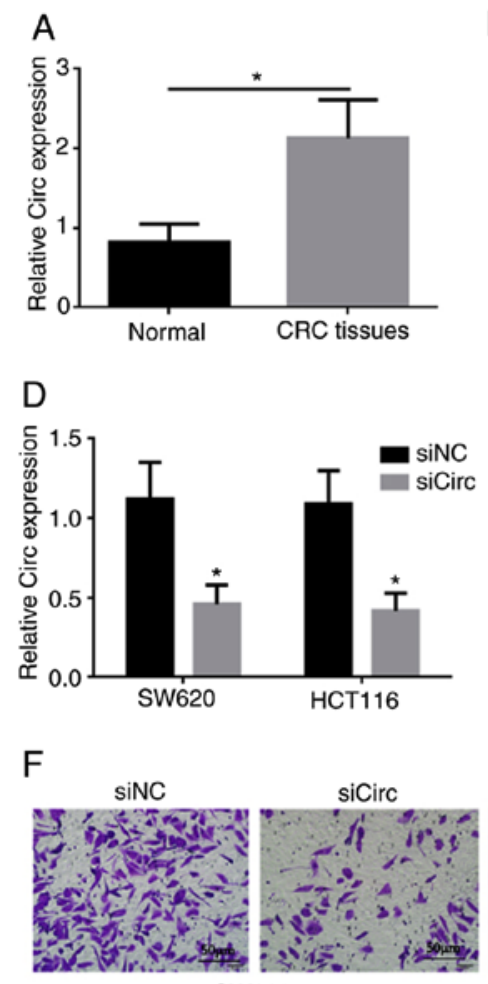

SW620
B
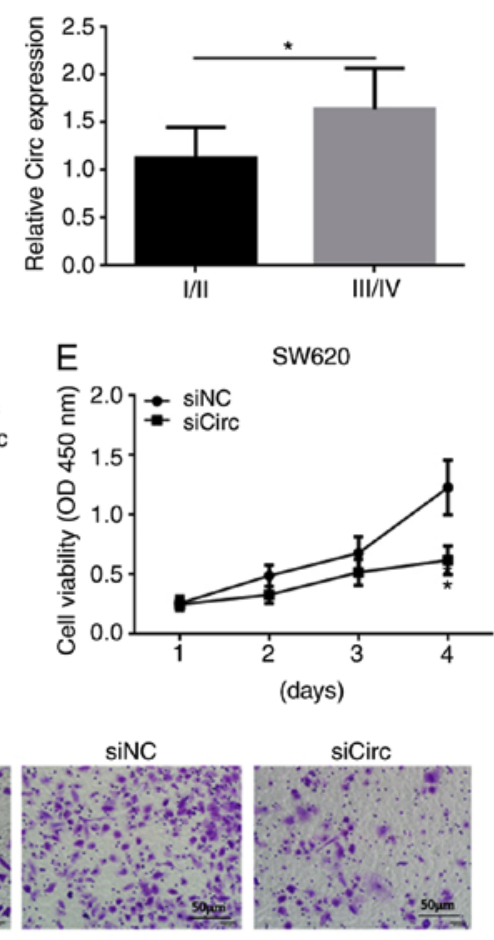

HCT116
C
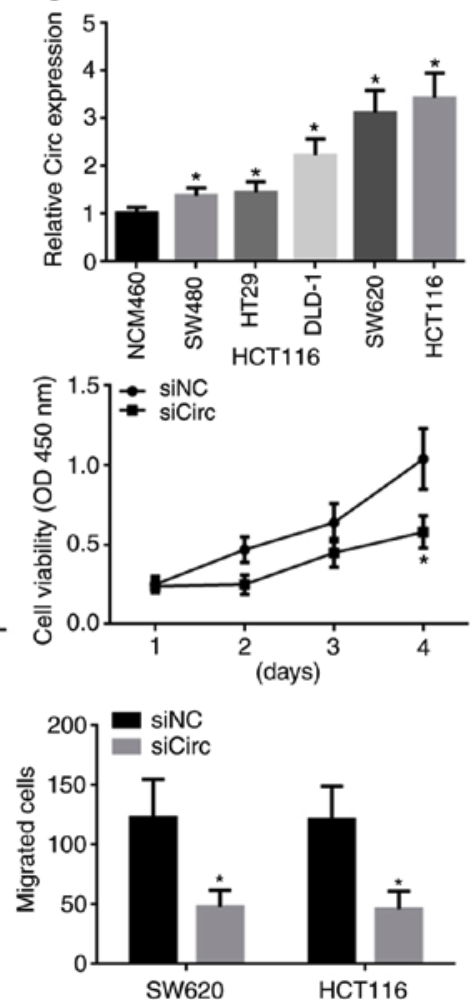

Figure 1. Downregulation of hsa_circ_0038646 inhibits human CRC cell proliferation and migration. (A) Relative hsa_circ_0038646 mRNA expression in human CRC tissues. (B) Relative hsa_circ_0038646 expression in different grades of CRC tumor tissues. (C) Relative hsa_circ_0038646 mRNA expression in human CRC cell lines. (D) Hsa_circ_0038646 mRNA expression in SW620 and HCT116 cells following transfection with siCirc. (E) Effect of hsa_circ_0038646 on SW620 and HCT116 cell proliferation as detected by CCK-8 assays. (F) Role of hsa_circ_0007534 in cell migration as determined by Transwell assays. ${ }^{*} \mathrm{P}<0.05$ vs. NCM460 cells and siNC group, respectively. All experiments were performed in triplicate. CRC, colorectal cancer; $\mathrm{NC}$, negative control; si, small interfering; Circ, hsa_circ_0038646; OD, optical density.

Biosystems; Thermo Fisher Scientific, Inc.). The following primer sequences (Generay Biotech Co., Ltd.) were used for qPCR: hsa_circ_0038646 forward, 5'-TATGGTGGAGAA GCGGGTGTT-3' and reverse, 5'-CCAGGGCAGGAGAAT GTGA-3'; GRIK3 forward, 5'-GCTGGTCTGCACTGAACT CT-3' and reverse, 5'-AAAGGGCATCCCCTGAATGG-3'; GAPDH forward, 5'-AGGTGAAGGTCGGAGTCAAC-3' and reverse, 5'-CGCTCCTGGAAGATGGTGAT-3'; miR-331-3p forward, 5'-GAGCTGAAAGCACTCCCAA-3' and reverse, 5'-CACACTCTTGATGTTCCAGGA-3'; and U6 forward, 5'-AGAGCCTGTGGTGTCCG-3' and reverse, 5'-CATCTT CAAAGCACTTCCCT-3'. The following thermocycling conditions were used for qPCR: Initial denaturation at $95^{\circ} \mathrm{C}$ for $1 \mathrm{~min}$, followed by 28 cycles of denaturation at $95^{\circ} \mathrm{C}$ for $10 \mathrm{sec}$, annealing at $58^{\circ} \mathrm{C}$ for $15 \mathrm{sec}$, elongation at $72^{\circ} \mathrm{C}$ for $5 \mathrm{sec}$ and a final extension at $72^{\circ} \mathrm{C}$ for $5 \mathrm{sec}$. GAPDH and human U6 RNA were used as internal controls for quantification of hsa_circ_0038646 and GRIK3, and miR-331-3p, respectively. The $2^{-\Delta \Delta C q}$ method (34) was used for quantification and a 2-fold change was considered significant. All RT-qPCR assays were performed in triplicate. Furthermore, the correlation between expression of hsa_circ_0038646 and miR-331-3p, or between the expression of miR-331-3p and GRIK3 was calculated.

Western blotting. Total protein was obtained from CRC cells with RIPA lysis buffer (Beyotime Institute of Biotechnology) according to the manufacturer's instructions. Protein concentrations were determined using a BCA protein assay kit (Beyotime Institute of Biotechnology). Proteins (40 $\mu \mathrm{g})$ were isolated via $10 \%$ SDS-PAGE and transferred to PVDF membranes (EMD Millipore). After blocking with 5\% milk prepared in Tris-buffered saline with $0.05 \%$ Tween-20 (TBST) at room temperature for $1 \mathrm{~h}$, membranes were incubated at $4{ }^{\circ} \mathrm{C}$ overnight with primary antibodies against GRIK3 (1:1,000; cat. no. ab183035; Abcam) and GAPDH (1:2,500; cat. no. ab9845; Abcam). The membranes were rinsed four times with TBST. A secondary antibody conjugated to horseradish peroxidase (1:2,000; cat. no. ab6721; Abcam) was added, and the membranes were incubated for $1 \mathrm{~h}$ at room temperature. Protein bands were visualized using the enhanced chemiluminescence kit (EMD Millipore). Image Lab Software (version 2.0; Bio-Rad Laboratories, Inc.) in the ChemiDoc XRS System (Thermo Fisher Scientific, Inc.) was used for quantification.

Luciferase reporter assay. Bioinformatics software Circular RNA Interactome (https://circinteractome.nia.nih.gov/index. html) was used to predict the presence of binding sites for miR-331-3p in hsa_circ_0038646 and GRIK3, and a luciferase reporter assay was performed to validate the association between hsa_circ_0038646, miR-331-3p and GRIK3. SW620 and HCT116 cells were seeded into 24-well plates at the density of $5 \times 10^{4}$ and co-transfected with wild-type (WT) or mutated (Mut) hsa_circ_0038646, which were inserted into the reporter vector pmirGLO (600 ng; Promega Corporation) 
A

hsa_circ_0038646 (5'...3') GUAUGGACUCAUCCACCAGGGGA

hsa-miR-331-3p (3'...5')

AAGAUCCUAUCCGGGUCCCCG

hsa_circ_0038646 (5'...3') GUAUGGACUCAUCCAGGUCCCCA

Mutation
B

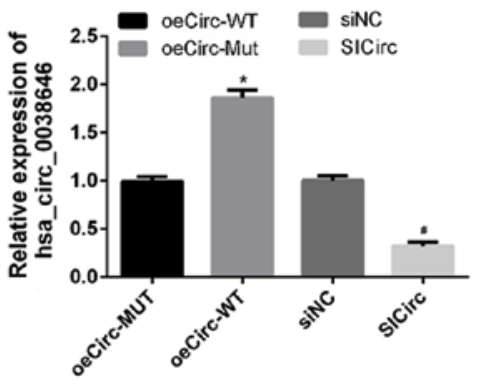

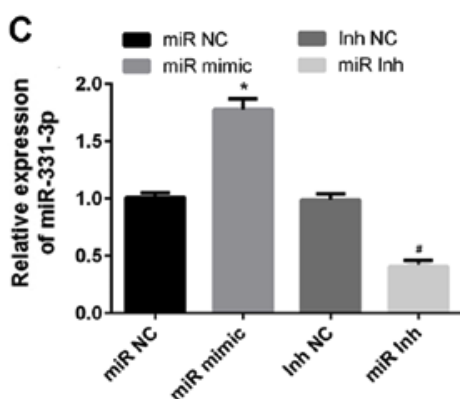

$\mathbf{F}$

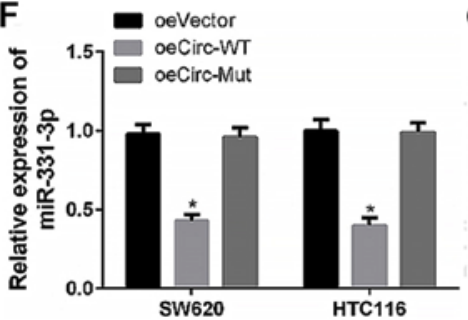

D

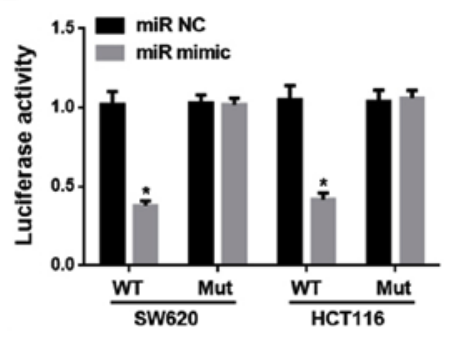

G

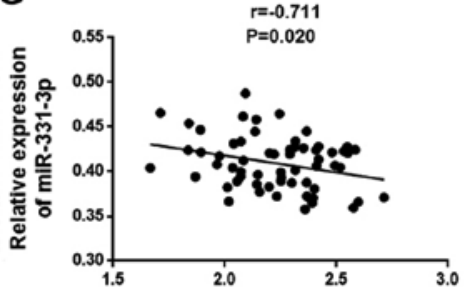

E

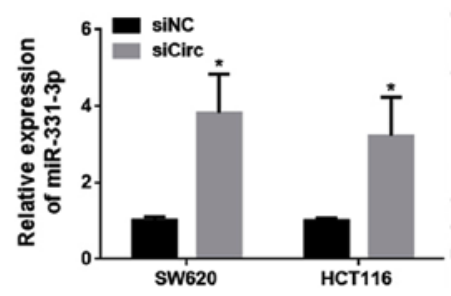

H

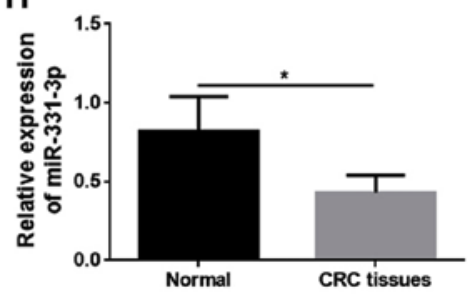

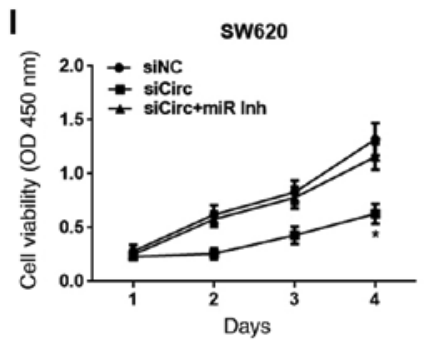
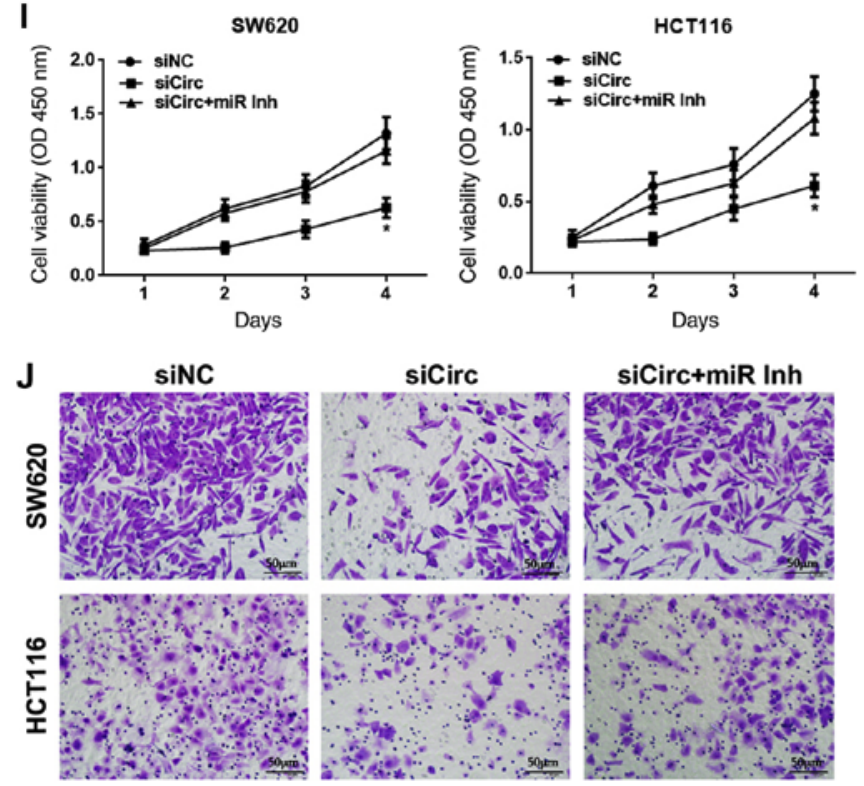

siCirc+miR Inh
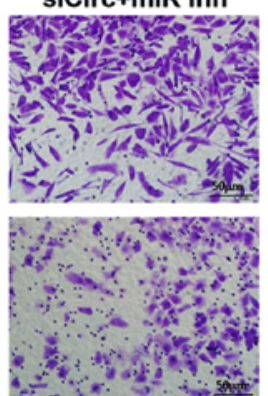

Figure 2. Hsa_circ_0038646 modulates cell proliferation and migration via sponging of miR-331-3p. (A) Predicted binding site between hsa_circ_0038646 and miR-331-3p. (B) Expression of hsa_circ_0038646 after transfection with oeCirc or siCirc. (C) Overexpression or inhibition of miR-331-3p by mimics or inhibitors, respectively. (D) miR-331-3p is a target of hsa_circ_0038646, as shown by dual-luciferase reporter assays in SW620 and HCT116 cells. (E) Hsa circ_0038646 negatively regulated miR-331-3p expression in vitro. (F) Overexpression of WT hsa_circ_0038646 inhibited miR-331-3p expression in CRC cells compared to oeVector group. (G) Relationship between hsa_circ_0038646 and miR-331-3p expression in CRC tissues. (H) Relative miR-331-3p mRNA expression in CRC tissues. Inhibition of miR-331-3p rescued the suppressed CRC cell. (I) proliferation and (J) migration mediated by hsa_circ_0038646 knockdown. "P $<0.05$ vs. oeCirc-Mut group, miR NC, oeVector group and siNC group, respectively; ${ }^{\#} \mathrm{P}<0.05$ vs. siNC group and Inh NC group, respectively. All experiments were performed in triplicate. CRC, colorectal cancer; NC, negative control; si, small interfering; miR, miR-331-3p; WT, wild-type; Mut, mutated; oe, overexpression vector; Inh, inhibitor; OD, optical density.

and GRIK3 3'-UTR reporter plasmid sequences, which were inserted into the pGL3 vector (600 ng; Shanghai GenePharma, Co., Ltd.) with either miR-331-3p mimics or NC (120 nM), using Lipofectamine ${ }^{\circledR} 2000$ transfection reagent (Invitrogen; Thermo Fisher Scientific, Inc.). After 24 h of transfection, luciferase activity was measured with the Dual Luciferase 

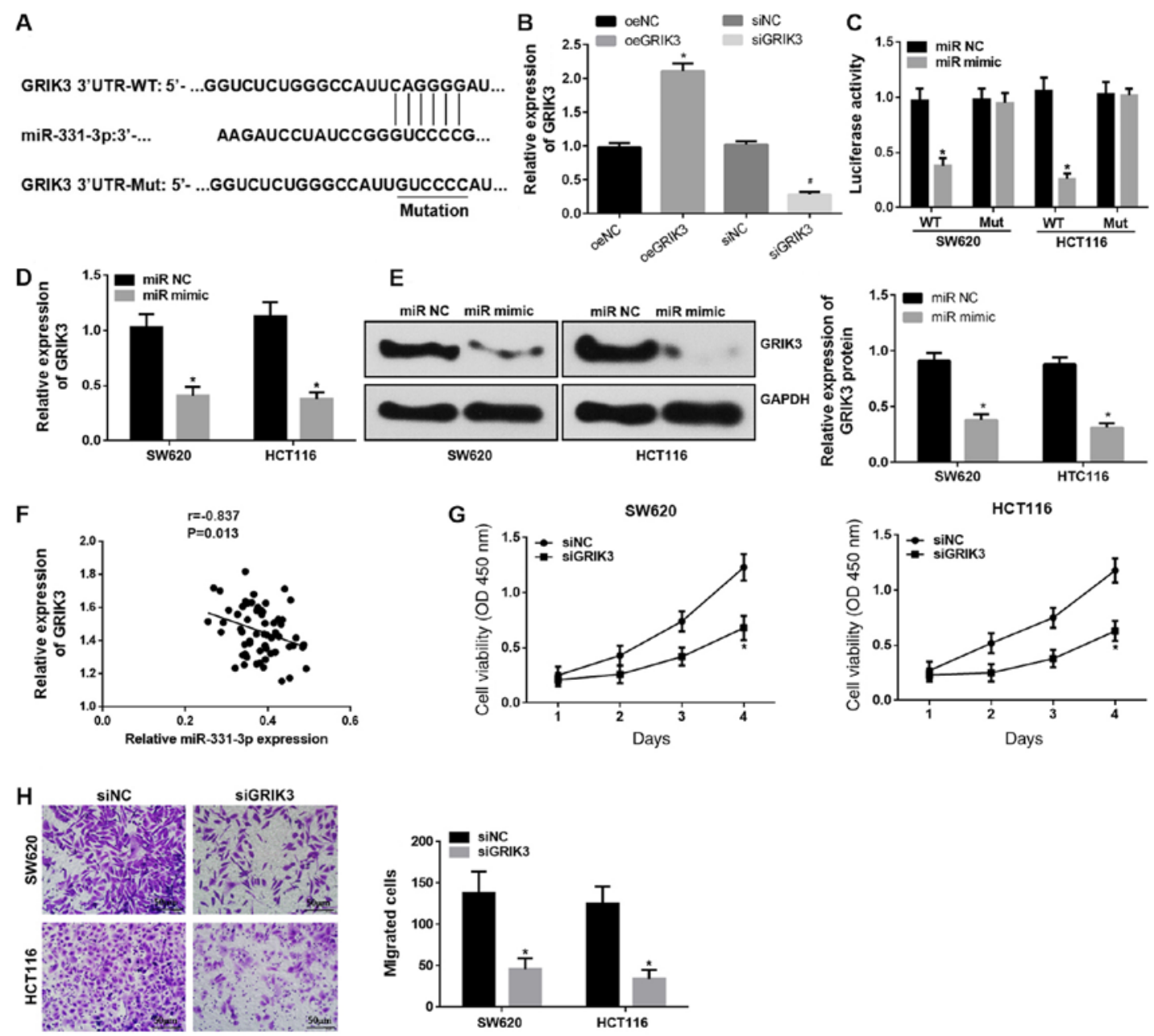

Figure 3. GRIK3 is a target of miR-331-3p. (A) Predicted binding site between miR-331-3p and GRIK3. (B) Expression of GRIK3 following transfection with oeGRIK3 or siRNA. (C) GRIK3 is a target of miR-331-3p, as shown by dual-luciferase reporter assay in vitro. (D) RT-qPCR and (E) western blotting show that upregulation of miR-331-3p repressed GRIK3 mRNA and protein levels in human CRC cells, respectively. (F) Association between miR-331-3p and GRIK3 expression in CRC tissues. Roles of GRIK3 in CRC cell $(\mathrm{G})$ proliferation and $(\mathrm{H})$ migration. " $\mathrm{P}<0.05$ vs. oeNC group, miR NC and siNC group, respectively; ${ }^{\#} \mathrm{P}<0.05$ vs. siNC group. All experiments were performed in triplicate. CRC, colorectal cancer; NC, negative control; si, small interfering; miR, miR-331-3p; WT, wild-type; Mut, mutated; oe, overexpression vector; OD, optical density; GRIK3, glutamate receptor ionotropic kainate 3.

Reporter Assay System (Promega Corporation) and normalized to Renilla luciferase activity.

Statistical analysis. Data were analyzed with SPSS 16.0 software (SPSS, Inc.) and presented as the mean \pm standard deviation. Student's t-tests and one-way ANOVAs followed by Tukey's test were used for the analysis of differences between two and multiple groups, respectively. Correlation analysis was conducted using Pearson correlation test where appropriate. Statistical significance was considered as $\mathrm{P}<0.05$. All experiments were performed in triplicate.

\section{Results}

Expression and function of hsa_circ_0038646 in human CRC.Hsa_circ_0038646 exhibited increased expression in human CRC tissue compared to normal tissue (Fig. 1A). This aberrant expression was positively associated with a higher tumor grade (III/IV) in CRC (Fig. 1B). Hsa_circ_0038646 also had increased expression, compared to a control cell line, in various human CRC cell lines, including SW480, HT29,
DLD-1, SW620 and HCT116, and was particularly highly expressed in SW620 and HCT116 cells (Fig. 1C). These findings indicated that increased hsa_circ_0038646 expression might be related to $\mathrm{CRC}$ progression.

SW620 and HCT116 cells with reduced expression of hsa_circ_0038646 were generated using siRNA targeting hsa_circ_0038646 (siCirc), and hsa_circ_0038646 expression levels were detected by RT-qPCR (Fig. 1D). Reduced expression of hsa_circ_0038646 reduced the proliferative capacity of SW620 and HCT116 cells, and showed a significant difference on day 4 of incubation as determined using a CCK-8 assay (Fig. 1E). Moreover, Transwell assays also revealed that reduced expression of hsa_circ_0038646 inhibited the migration of SW620 and HCT116 cells (Fig. 1F).

Hsa_circ_0038646 regulates CRC cell proliferation and migration by targeting miR-331-3p. Bioinformatics analysis predicted the presence of a binding site between hsa_circ_0038646 and miR-331-3p (Fig. 2A). However, the expression levels of hsa_circ_0038646 in CRC with low tumor grades (I and II) are not currently available in public 

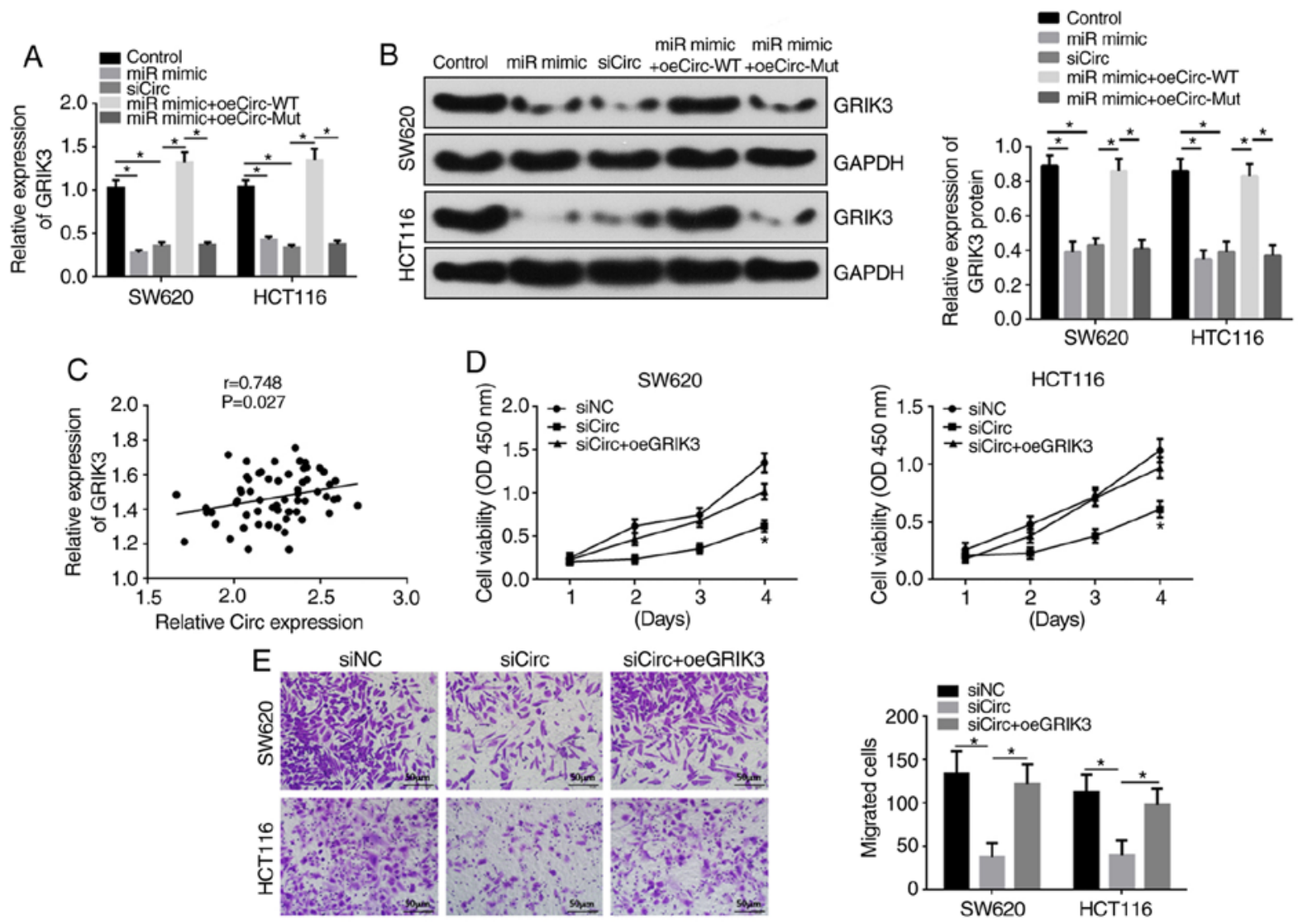

Figure 4. Hsa_circ_0038646 facilitates CRC cell proliferation and migration via upregulation of GRIK3 by sponging miR-331-3p. Relative GRIK3 (A) mRNA and (B) protein levels in SW620 and HCT116 cells transfected with targeted plasmids. (C) Relationship between hsa_circ_0038646 and GRIK3 expression in CRC tissues. Overexpression of GRIK3 rescued the suppressed CRC cell (D) proliferation and (E) migration mediated by hsa_circ_0038646 knockdown. ${ }^{*} \mathrm{P}<0.05$ vs. siNC group or siCirc group. All experiments were performed in triplicate. CRC, colorectal cancer; NC, negative control; si, small interfering; miR, miR-331-3p; WT, wild-type; Mut, mutated; oe, overexpression vector; OD, optical density; GRIK3, glutamate receptor ionotropic kainate 3.

databases. WT and Mut luciferase reporter plasmids were designed (Fig. 2A). Additionally, to validate the targeting relationship between hsa_circ_0038646 and miR-331-3p, oe of hsa_circ_0038646 was conducted using plasmids encoding WT or Mut hsa_circ_0038646 cDNA (oeCirc-WT and oeCirc-Mut, respectively), with hsa_circ_0038646 upregulated in cells transfected with the WT vector compared with the Mut vector (Fig. 2B). Subsequently, miR-331-3p expression was upregulated or inhibited via transfection with miR-331-3p mimics or inhibitors, respectively (Fig. 2C). It was then found that upregulation of miR-331-3p reduced the luciferase activity in SW620 and HCT116 cells transfected with the WT reporter plasmid. Interestingly, no effect was observed in the luciferase reporter assay with cells transfected with the Mut plasmid (Fig. 2D). RT-qPCR and Pearson correlation analysis also indirectly indicated that miR-331-3p is a target of hsa_circ_0038646 (Fig. 2E-G). Downregulation of hsa_circ_0038646 expression resulted in a significant increase in miR-331-3p levels in SW620 and HCT116 cells (Fig. 2E). In addition, compared with the oeVector group, the expression of miR-331-3p was significantly suppressed by oeCirc-WT, but not by oeCirc-Mut (Fig. 2F). Pearson correlation analysis showed that miR-331-3p levels were also negatively correlated with hsa_circ_0038646 levels in human CRC tissues (Fig. 2G).

RT-qPCR revealed that miR-331-3p was downregulated in human CRC tissues (Fig. 2H). Reduced expression of hsa_circ_0038646resultedin reduced humanCRC cell proliferation and migration in vitro. However, following co-transfection with both siCirc and miR-331-3p inhibitors, there was no reduction in cell proliferation and migration, indicating that decreased expression of miR-331-3p attenuated the effects of hsa_circ_0038646 siRNA (Fig. 2I and J).

GRIK3 is a targetofmiR-331-3p. Bioinformatics analysis revealed that GRIK3 contains a binding site for miR-331-3p (Fig. 3A). GRIK3 was upregulated or downregulated using oeGRIK3 or siRNA, respectively (Fig. 3B). The luciferase reporter assays were then repeated, and this confirmed that transfection of miR-331-3p mimics decreased the luciferase activity of SW620 and HCT116 cells transfected with the WT-GRIK3-3'-UTR, but had no effect in SW620 and HCT116 cells transfected with the Mut-GRIK3-3'-UTR (Fig. 3C). RT-qPCR and western blotting analyses revealed that upregulation of miR-331-3p decreased GRIK3 mRNA and protein levels (Fig. 3D and E). GRIK3 levels were inversely correlated with miR-331-3p in CRC tissues (Fig. 3F). To the author's best knowledge, the present study has shown the first evidence suggesting that the downregulation of GRIK3 expression significantly inhibits SW620 and HCT116 cell proliferation and migration (Fig. $3 \mathrm{G}$ and $\mathrm{H}$ ).

Hsa_circ_0038646 accelerates human CRC cell proliferation and migration by upregulating GRIK3 via sponging of 
miR-331-3p. Compared to the control group, miR-331-3p upregulation or hsa_circ_0038646 knockdown decreased mRNA and protein levels of GRIK3 in SW620 and HCT116 cells, as shown by RT-qPCR and western blotting (Fig. 4A and B). Furthermore, transfection with oeCirc-WT, but not oeCirc-Mut, abolished the effects of miR-331-3p mimics on GRIK3 levels compared with the siCirc group. GRIK3 expression was also positively correlated with hsa_circ_0038646 in human CRC tissues (Fig. 4C).

CCK-8 and Transwell assays showed that compared to the siNC group, cell proliferation and migration were significantly decreased in the siCirc group, whilst oeGRIK3 markedly rescued the suppression effect of siCirc on SW620 and HCT116 cell proliferation and migration (Fig. 4D and E).

\section{Discussion}

CRC is a fatal illness with high mortality and morbidity (35), and high rates of metastasis and recurrence (6). Despite the use of surgical and adjuvant therapy, the survival rate has not improved dramatically, and $~ 50 \%$ of patients die from local recurrence or metastasis following surgery (36). Novel biomarkers and effective therapeutic targets are urgently required. A single report has demonstrated that miR-331-3p is significantly downregulated in both human CRC cells and tissues (28). This suppression of miR-331-3p expression accelerated cell proliferation and inhibited apoptosis (14). miR-331-3p has also been reported to be downregulated in cervical cancer (CC) (37). Upregulation of miR-331-3p restrained migration and invasion, and facilitated apoptosis in $\mathrm{CC}$ cells (28). Downregulation of miR-331-3p has also been shown to inhibit apoptosis and promote cell proliferation in human papillomavirus-positive CC (38), as well as facilitate proliferation, invasion and migration in gastric cancer (39). The role of miR-331-3p in CRC cell migration is unclear, and the potential up- and downstream regulatory mechanisms of miR-331-3p are unknown. In the present study, it was shown that miR-331-3p was downregulated in human CRC tissues, and reduced expression of miR-331-3p resulted in increased cell proliferation. Reduced expression of miR-331-3p also inhibited cell migration in human CRC cells. It was predicted using bioinformatic analysis that hsa_circ_0038646 and GRIK3 contain miR-331-3p binding sites, and may be up- and downstream of miR-331-3p, respectively. Based on this, it was hypothesized that the hsa_circ_0038646/miR-331-3p/GRIK3 axis may play a pivotal role in CRC progression.

The expression and functions of hsa_circ_0038646 in CRC tissues and cells were assessed by RT-qPCR, and CCK- 8 and Transwell assays, respectively. To the author's knowledge, there has been no report on the role of hsa_circ_0038646 in any disease. It was determined in the present study that hsa_circ_0038646 exhibited increased expression compared to controls in human CRC tissues, as well as various human CRC cell lines (SW480, HT29, DLD-1, SW620 and HCT116). Hsa_circ_0038646 was especially highly expressed in SW620 and HCT116 cells. This aberrant hsa_circ_0038646 expression was positively associated with higher tumor grade (III/IV) in CRC tissues. Next, SW620 and HCT116 cells with reduced expression of hsa_circ_0038646 were constructed. Decreased expression of hsa_circ_0038646 inhibited CRC cell proliferation and migration. These data indicated that hsa_circ_0038646 acts as an oncogene in CRC progression. Previous reports have shown that circRNAs are differentially expressed in various cancers, including CRC (39). Increasing evidence has indicated that circRNAs modulate multiple physiological and pathological processes, including cell survival, proliferation, metastasis, tumorigenesis and tumor progression, and may be significant biomarkers in cancers $(40,41)$. The present study demonstrated that hsa_circ_0038646 is upregulated in CRC, which may result in increased cell proliferation and migration.

CircRNAs exert their functions by acting as miRNA sponges (42). To further confirm whether hsa_circ_0038646 exerts its role in CRC via sponging of miR-331-3p, hsa_circ_0038646 reporter plasmids sequences were generated containing the WT and Mut 3'-UTR, according to the predicted binding site. Using luciferase reporter assays and RT-qPCR, it was found that miR-331-3p was a potential target of hsa_circ_0038646, and that hsa_circ_0038646 could negatively regulate miR-331-3p expression. Furthermore, CCK-8 and Transwell assays suggested that suppression of miR-331-3p inhibits the effects of reduced hsa_circ_0038646 expression on CRC cell proliferation and migration. These outcomes indicated that miR-331-3p functions as a tumor inhibitor in CRC. The present study is the first to report that hsa_circ_0038646 exerts its functions in CRC via sponging of miR-331-3p.

miRNAs play roles in cancer by transcriptionally and post-transcriptionally suppressing the expression of target genes via complementation of the 3'-UTR of mRNAs (43). In the present study, a downstream target of miR-331-3p was investigated. GRIK3 was identified as a target of miR-331-3p in vitro, and inhibition of GRIK3 repressed CRC cell proliferation and migration. GRIK3 has been reported to function as an oncogenic protein, and has served as a prognostic and therapeutic target in several cancers, including glioma (44), lung adenocarcinoma (45), gastric cancer (31) and breast cancer (30). To the author's best knowledge, the role of GRIK3 in CRC has not been reported, and the present study is the first to demonstrate that GRIK3 functions as an oncogenic protein in CRC.

In the present study, RT-qPCR and western blotting were used to determine that GRIK3 expression was modulated by hsa_circ_0038646 in human SW620 and HCT116 cells. This demonstrated that hsa_circ_0038646 accelerates human CRC cell proliferation and migration by upregulating GRIK3 via sponging of miR-331-3p.

Overall, the present study suggested that hsa_circ_0007534 and GRIK3 act as tumor promoters in CRC progression, and miR-331-3p can inhibit human CRC cell migration. This demonstrates that the hsa_circ_0007534/miR-331-3p/GRIK3 axis may be a promising therapeutic target for CRC.

\section{Acknowledgements}

Not applicable.

\section{Funding}

No funding was received. 


\section{Availability of data and materials}

The datasets used and/or analyzed during the current study are available from the corresponding author on reasonable request.

\section{Authors' contributions}

HD and JZ made substantial contributions to the conception of the study, designing the experiments, interpretation of the data and writing the paper. $\mathrm{ZH}$ and $\mathrm{FF}$ performed the experiments and collected data. DC, LZ and EH acquired and analyzed the data. JB and HW analyzed and interpreted the data. All authors read and approved the final manuscript.

\section{Ethics approval and consent to participate}

The present study was approved by the Clinical Ethical Committee of Tianjin Baodi Hospital (approval no. 2018-6), and performed in accordance with The Declaration of Helsinki. Written informed consent was provided by all participants prior to the study start.

\section{Patients consent for publication}

Not applicable.

\section{Competing interests}

The authors declare that they have no competing interests.

\section{References}

1. Lipton LR: Familial colorectal cancer and polyposis genes, pathways and predictions (unpublished $\mathrm{PhD}$ thesis). University of London, 2006.

2. Kahouli I, Tomaro-Duchesneau C and Prakash S: Probiotics in colorectal cancer (CRC) with emphasis on mechanisms of action and current perspectives. J Med Microbiol 62: 1107-1123, 2013.

3. Siegel R, Desantis C and Jemal A: Colorectal cancer statistics, 2014. CA Cancer J Clin 64: 104-117, 2014.

4. Jemal A, Bray F, Center MM, Ferlay J, Ward E and Forman D: Global cancer statistics. CA Cancer J Clin 61: 69-90, 2011.

5. Chua YJ and Zalcberg JR: Progress and challenges in the adjuvant treatment of stage II and III colon cancers. Expert Rev Anticancer Ther 8: 595-604, 2008.

6. Hamilton SR and Aaltonen LA (eds): Pathology and genetics. Tumours of the digestive system. In: WHO Classification of Tumours. Vol 2. IARC Press, Lyon, pp103-119, 2000.

7. Wei W, Yang Y, Cai J, Cui K, Li RX, Wang H, Shang X and Wei D: miR-30a-5p suppresses tumor metastasis of human colorectal cancer by targeting ITGB3. Cell Physiol Biochem 39: $1165-1176,2016$.

8. Croce CM: Causes and consequences of microRNA dysregulation in cancer. Nat Rev Genet 10: 704-714, 2009.

9. Singh SR and Rameshwar P (eds): MicroRNA in development and in the progression of cancer. Springer, New York, NY, 2014.

10. Ryan BM, Robles AI and Harris CC: Genetic variation in microRNA networks: The implications for cancer research. Nat Rev Cancer 10: 389-402, 2010.

11. Bartel DP: MicroRNAs: Genomics, biogenesis, mechanism, and function. Cell 116: 281-297, 2004.

12. Wang L, Tang H, Thayanithy V, Subramanian S, Oberg AL, Cunningham JM, Cerhan JR, Steer CJ and Thibodeau SN: Gene networks and microRNAs implicated in aggressive prostate cancer. Cancer Res 69: 9490-9497, 2009.

13. Zhao D, Sui Y and Zheng X: MiR-331-3p inhibits proliferation and promotes apoptosis by targeting HER2 through the $\mathrm{PI} 3 \mathrm{~K} / \mathrm{Akt}$ and ERK1/2 pathways in colorectal cancer. Oncol Rep 35: 1075-1082, 2016.
14. Zanette DL, Rivadavia F, Molfetta GA, Barbuzano FG, Proto-Siqueira R, Silva WA Jr, Falcão RP and Zago MA: miRNA expression profiles in chronic lymphocytic and acute lymphocytic leukemia. Braz J Med Biol Res 40: 1435-1440, 2007.

15. Nymark P, Guled M, Borze I, Faisal A, Lahti L, Salmenkivi K, Kettunen E, Anttila $S$ and Knuutila S: Integrative analysis of microRNA, mRNA and aCGH data reveals asbestos- and histology-related changes in lung cancer. Genes Chromosomes Cancer 50: 585-597, 2011.

16. Epis MR, Giles KM, Candy PA, Webster RJ and Leedman PJ: miR-331-3p regulates expression of neuropilin-2 in glioblastoma. J Neurooncol 116: 67-75, 2014.

17. Guo X, Guo L, Ji J, Zhang J, Zhang J, Chen X, Cai Q, Li J, Gu Q, Liu B, et al: miRNA-331-3p directly targets E2F1 and induces growth arrest in human gastric cancer. Biochem Biophys Res Commun 398: 1-6, 2010.

18. Epis MR, Giles KM, Kalinowski FC, Barker A, Cohen RJ and Leedman PJ: Regulation of expression of deoxyhypusine hydroxylase $(\mathrm{DOHH})$, the enzyme that catalyzes the activation of eIF5A, by miR-331-3p and miR-642-5p in prostate cancer cells. J Biol Chem 287: 35251-35259, 2012.

19. Li X, Zhu J, Liu Y, Duan C, Chang $R$ and Zhang $C$ : MicroRNA-331-3p inhibits epithelial-mesenchymal transition by targeting ErbB2 and VAV2 through the Rac1/PAK1/ $\beta$-catenin axis in non-small-cell lung cancer. Cancer Sci 110: 1883-1896, 2019.

20. Chen X, Luo H, Li X, Tian X, Peng B, Liu S, Zhan T, Wan Y, Chen W, Li Y, et al: miR-331-3p functions as an oncogene by targeting ST7L in pancreatic cancer. Carcinogenesis 39: 1006-1015, 2018

21. Ebbesen KK, Kjems J and Hansen TB: Circular RNAs: Identification, biogenesis and function. Biochim Biophys Acta 1859: 163-168, 2016.

22. Zhong Y, Du Y, Yang X, Mo Y, Fan C, Xiong F, Ren D, Ye X, Li C, Wang Y, et al: Circular RNAs function as ceRNAs to regulate and control human cancer progression. Mol Cancer 17: 79, 2018.

23. Salzman J: Circular RNA expression: Its potential regulation and function. Trends Genet 32: 309-316, 2016.

24. Ge J, Jin Y, Lv X, Liao Q, Luo C, Ye G and Zhang X: Expression profiles of circular RNAs in human colorectal cancer based on RNA deep sequencing. J Clin Lab Anal 33: e22952, 2019.

25. Zhao ZJ and Shen J: Circular RNA participates in the carcinogenesis and the malignant behavior of cancer. RNA Biol 14: 514-521, 2017.

26. Zhang L, Song X, Chen X, Wang Q, Zheng X, Wu C and Jiang J: Circular RNA CircCACTIN promotes gastric cancer progression by sponging MiR-331-3p and regulating TGFBR1 expression. Int J Biol Sci 15: 1091-1103, 2019.

27. Chen HH,Zong J and Wang SJ: LncRNA GAPLINC promotes the growth and metastasis of glioblastoma by sponging miR-331-3p. Eur Rev Med Pharmacol Sci 23: 262-270, 2019.

28. Luan X and Wang Y: LncRNA XLOC_006390 facilitates cervical cancer tumorigenesis and metastasis as a ceRNA against miR-331-3p and miR-338-3p. J Gynecol Oncol 29: e95, 2018.

29. Liu T, Song Z and Gai Y: Circular RNA circ_0001649 acts as a prognostic biomarker and inhibits NSCLC progression via sponging miR-331-3p and miR-338-5p. Biochem Biophys Res Commun 503: 1503-1509, 2018.

30. Xiao B, Kuang Z, Zhang W, Hang J, Chen L, Lei T, He Y, Deng C, $\mathrm{Li} \mathrm{W}, \mathrm{Lu} \mathrm{J}$, et al: Glutamate ionotropic receptor kainate type subunit 3 (GRIK3) promotes epithelial-mesenchymal transition in breast cancer cells by regulating SPDEF/CDH1 signaling. Mol Carcinog 58: 1314-1323, 2019.

31. Gong B, Li Y, Cheng Z, Wang P, Luo L, Huang H, Duan S and Liu F: GRIK3: A novel oncogenic protein related to tumor TNM stage, lymph node metastasis, and poor prognosis of GC. Tumour Biol 39: 1010428317704364, 2017.

32. Novák $J$ and Fabian P: Comments on the TNM classification of malignant tumours-7th edition. Klin Onkol 24: 149-150, 2011 (In Czech)

33. Livak KJ and Schmittgen TD: Analysis of relative gene expression data using real-time quantitative PCR and the 2(-Delta Delta C(T)) method. Methods 25: 402-408, 2001.

34. Li GF, Li L, Yao ZQ and Zhuang SJ: Hsa circ_0007534/miR-761/ZIC5 regulatory loop modulates the proliferation and migration of glioma cells. Biochem Biophys Res Commun 499: 765-771, 2018.

35. Yin Q, Wang PP, Peng R and Zhou H: MiR-19a enhances cell proliferation, migration, and invasiveness through enhancing lymphangiogenesis by targeting thrombospondin-1 in colorectal cancer. Biochem Cell Biol 97: 731-739, 2019. 
36. Zhang H, Wang R and Wang M: miR-331-3p suppresses cell invasion and migration in colorectal carcinoma by directly targeting NRP2. Oncol Lett 18: 6501-6508, 2019.

37. Arnold M, Sierra MS, Laversanne M, Soerjomataram I, Jemal A and Bray F: Global patterns and trends in colorectal cancer incidence and mortality. Gut 66: 683-691, 2017.

38. Zhang M, Song Y and Zhai F: ARFHPV E7 oncogene, lncRNA HOTAIR, miR-331-3p and its target, NRP2, form a negative feedback loop to regulate the apoptosis in the tumorigenesis in HPV positive cervical cancer. J Cell Biochem 119: 4397-4407, 2018.

39. Jiang W, Zhang X, Chu Q, Lu S, Zhou L, Lu X, Liu C, Mao L, Ye C, Timko MP, et al: The circular RNA profiles of colorectal tumor metastatic cells. Front Genet 9: 34, 2018.

40. Huang WJ, Wang Y, Liu S, Yang J, Guo SX, Wang L, Wang H and Fan YF: Silencing circular RNA hsa_circ_0000977 suppresses pancreatic ductal adenocarcinoma progression by stimulating miR-874-3p and inhibiting PLK1 expression. Cancer Lett 422 70-80, 2018

41. Zhang S, Zeng X, Ding T, Guo L, Li Y, Ou S and Yuan H: Microarray profile of circular RNAs identifies hsa_circ_0014130 as a new circular RNA biomarker in non-small cell lung cancer. Sci Rep 8: 2878, 2018
42. Li G, Yang H, Han K, Zhu D, Lun P and Zhao Y: A novel circular RNA, hsa_circ_0046701, promotes carcinogenesis by increasing the expression of miR-142-3p target ITGB8 in glioma. Biochem Biophys Res Commun 498: 254-261, 2018.

43. Chen K and Rajewsky N: The evolution of gene regulation by transcription factors and microRNAs. Nat Rev Genet 8: 93-103, 2007.

44. Yuan Y, Xiang W, Yanhui L, Ruofei L, Yunhe M, Jiewen L and Qing M: Dysregulation of microRNA-128 expression in WHO grades 2 glioma is associated with glioma-associated epilepsy: Down-regulation of miR-128 induces glioma-associated seizure. Epilepsy Res 127: 6-11, 2016.

45. Pradhan MP, Desai A and Palakal MJ: Systems biology approach to stage-wise characterization of epigenetic genes in lung adenocarcinoma. BMC Syst Biol 7: 141, 2013.

This work is licensed under a Creative Commons Attribution-NonCommercial-NoDerivatives 4.0 International (CC BY-NC-ND 4.0) License. 